\title{
Promoting effect of lactoferrin on barrier function and epithelial differentiation of human keratinocytes
}

\begin{tabular}{|r|l|}
\hline Journal: & Biochemistry and Cell Biology \\
\hline Manuscript ID & bcb-2016-0147.R2 \\
\hline Manuscript Type: & Article \\
\hline Complete List of Authors: & $\begin{array}{r}\text { Uchida, Ryo; Tsukuba Daigaku, Graduate School of Life and Environmental } \\
\text { Sciences; National Agriculture and Food Research Organization, National } \\
\text { Institute of Livestock and Grassland Science } \\
\text { Aoki, Reiji; National Agriculture and Food Research Organization, National } \\
\text { Institute of Livestock and Grassland Science } \\
\text { Aoki-Yoshida, Ayako; The University of Tokyo, Graduate School of } \\
\text { Agricultural and Life Sciences; National Agriculture and Food Research } \\
\text { Organization, National Institute of Livestock and Grassland Science } \\
\text { Tajima, Atsushi; University of Tsukuba, Faculty of Life and Environmental } \\
\text { Sciences } \\
\text { Takayama, Yoshiharu; National Agriculture and Food Research } \\
\text { Organization, National Institute of Livestock and Grassland Science }\end{array}$ \\
\hline Keyword: & $\begin{array}{l}\text { involucrin, filaggrin, sterol regulatory element-binding protein-1, } \\
\text { transepithelial electrical resistance, p38 mitogen-activated protein kinase }\end{array}$ \\
\hline & \\
\hline
\end{tabular}


1 Promoting effect of lactoferrin on barrier function and epithelial differentiation of 2 human keratinocytes

4 Ryo Uchida ${ }^{\mathrm{a}, \mathrm{b}}$, Reiji Aoki ${ }^{\mathrm{a}}$, Ayako Aoki-Yoshida ${ }^{\mathrm{a}, \mathrm{c}}$, Atsushi Tajima ${ }^{\mathrm{b}}$, and Yoshiharu

5 Takayama ${ }^{\mathrm{a} *}$

6

7 a: Functional Biomolecules Research Group, National Institute of Livestock and 8 Grassland Science, National Agriculture and Food Research Organization (NARO)

9 b: Graduate School of Life and Environmental Sciences, University of Tsukuba

10 c: Department of Applied Biological Chemistry, Graduate School of Agricultural and 11 Life Sciences, The University of Tokyo

*Corresponding author: Yoshiharu Takayama

14 Functional Biomolecules Research Group, National Institute of Livestock and 15 Grassland Science, National Agriculture and Food Research Organization (NARO)

162 Ikenodai, Tsukuba, Ibaraki, 305-0901 Japan

17 Tel.: +81-29-838-8687, Fax: +81-29-838-8606, E-mail: takay@affrc.go.jp

\section{Abbreviations}

22 DMEM, Dulbecco's Modified Eagle's Medium; LRP-1, low-density lipoprotein 23 receptor-related protein-1; MEK, mitogen-activated protein kinase kinase; PVDF, 24 polyvinylidene difluoride; SREBP1, sterol regulatory element-binding protein-1; TER, 25 transepithelial electrical resistance; MAPK, Mitogen-activated protein kinase; PI-3K 26 Phosphoinositide 3-kinase; NHEK, normal human epipermal keratinocytes 


\section{Abstract}

2 The purpose of this study was to elucidate the effects of bovine lactoferrin on

3 keratinocyte differentiation and barrier function. Addition of bovine lactoferrin to

4 differentiating HaCaT human keratinocytes led to increased transepithelial electrical

5 resistance (TER), a marker of epithelial barrier function. This elevation was followed by

6 upregulation of two differentiation markers, involucrin and filaggrin. The expression

7 level of sterol regulatory element-binding protein-1 was also enhanced by bovine

8 lactoferrin. The lactoferrin-induced upregulation of involucrin and filaggrin expression

9 were confirmed in normal human epidermal keratinocytes (NHEK). Treatment with

10 SB203580, a p38 mitogen-activated protein kinase (MAPK) $\alpha$ inhibitor, impaired the

11 upregulation of involucrin and filaggrin expression in response to lactoferrin. The

12 elevation of p38 MAPK phosphorylation was further enhanced by lactoferrin in the

13 initial stage of differentiation of HaCaT keratinocytes. The findings suggest that bovine

14 lactoferrin promotes epithelial differentiation by a p38 MAPK-dependent mechanism.

16 Keywords: involucrin, filaggrin, sterol regulatory element-binding protein-1, 17 transepithelial electrical resistance, p38 mitogen-activated protein kinase

\section{Introduction}

22 The epidermis of the skin provides a protective barrier to the outside environment.

23 Skin wound healing is a critical process to restore barrier function and prevent infection

24 in response to disease or injury. Keratinocytes are a major cellular component of the 25 epidermis and are responsible for restoring the epidermis after injury by a process 26 termed re-epithelialization (Sivamani et al. 2007). The epidermis has a multilayered 27 structure and consists of four progressively differentiated layers. During 28 re-epithelialization, epidermal stem cells located in the basal layer of the epidermis 29 continue to proliferate, whereas the newly produced daughter cells start to differentiate, 30 migrating from the basal layer into the progressively differentiated layers, such as the 31 spinous layer, granular layer, and stratum corneum. This involves formation of the 32 cornified envelope, an insoluble complex of cross-linked proteins and lipids beneath the 
1 plasma membrane of keratinocytes. The cornified envelope plays an important role in 2 maintaining the structural integrity and barrier function of the epidermis. Involucrin and 3 filaggrin are markers of the terminal differentiation of keratinocytes. Involucrin, one of 4 the major protein components of the cornified envelope, is present in the upper layers of 5 spinous cells and is maintained in keratinocytes of the granular layer (Eckert et al. 2004). 6 Filaggrin is a structural protein specifically found in the granular layer and stratum 7 corneum. Sterol regulatory element-binding protein-1 (SREBP1) is a transcription 8 factor that regulates the expression of enzymes involved in fatty acid and cholesterol synthesis (Goldstein et al. 2006). Synthesis of fatty acids and cholesterol is required for 10 the barrier function of the epidermis. Earlier studies indicate that SREBP1 expression is 11 upregulated along with the differentiation of keratinocytes (Yokoyama et al. 2009). The 12 switch between keratinocyte growth and differentiation is tightly regulated. Loss of 13 proliferative potential is strictly associated with the differentiation of keratinocytes.

Lactoferrin is an iron-binding glycoprotein belonging to the transferrin family. It 15 can be detected in exocrine secretions of mammals such as milk, colostrum, tears, and 16 mucosal secretions. Lactoferrin contributes to host defense by its bactericidal and 17 immune-modulatory activities (Legrand et al. 2005; Actor et al. 2009). In addition, 18 recent studies indicate that topical administration of human lactoferrin increases the rate 19 of skin wound closure in normal and diabetic mice (Lyons et al. 2007; Engelmayer et al. 20 2008; Takayama and Aoki 2012). The promoting effect of human lactoferrin on wound 21 healing has been confirmed by clinical studies (Lyons et al. 2007). Lactoferrin promotes 22 the migration of epidermal keratinocytes and re-epithelialization of burn wounds in 23 swine (Tang et al. 2010; Takayama and Aoki 2012), suggesting that it promotes wound 24 healing by directly regulating epithelial keratinocytes.

25 We reported that bovine lactoferrin promotes the contractile activity of fibroblasts, 26 which play a critical role in wound healing by promoting wound contraction (Takayama 27 et al. 2003; Takayama and Aoki 2012). Furthermore, we found that bovine lactoferrin 28 promoted collagen and hyaluronan synthesis in human dermal fibroblasts, suggesting 29 that both human and bovine lactoferrin were effective for wound healing (Takayama 30 and Aoki 2012).

31 The purpose of this study was to investigate the effect of bovine lactoferrin on the 32 epithelial differentiation and barrier function of HaCaT keratinocytes. In this study, we 
1 used HaCaT human keratinocytes as a model of epithelial differentiation of 2 keratinocytes. Although $\mathrm{HaCaT}$ cells are immortalized cells, a similarity in the 3 calcium-induced induction of differentiation markers and the proliferation rate in non-differentiating conditions was observed between $\mathrm{HaCaT}$ cells and normal human keratinocytes (Micallef et al. 2009).

\section{Materials and methods}

8 Materials

Bovine lactoferrin was obtained from Fonterra (Auckland, New Zealand). The iron saturation of bovine lactoferrin was less than $30 \%,<20$ endotoxin units $(\mathrm{EU}) / \mathrm{mg}$, and $>95 \%$ purity. HaCaT cells were provided by Cell Lines Service (Eppelheim, Germany). Normal human epidermal keratinocytes (NHEK) were provided by Lifeline Cell

13 Technology (Frederick, MD, USA). High-glucose (4.5 g/ml) Dulbecco's Modified 14 Eagle's Medium (DMEM) was purchased from Sigma (St. Louis, MO, USA). 15 HuMedia-KG2 medium was purchased from Kurabo (Osaka, Japan). Penicillin and 16 streptomycin were obtained from Life Technologies (Carlsbad, CA, USA). CnT-07 17 keratinocyte growth medium and CnT-02 keratinocyte differentiation medium were 18 purchased from CELLnTEC (Bern, Switzerland). Cell culture inserts were obtained 19 from Corning (Corning, NY, USA). RIPA buffer was obtained from Nacalai Tesque 20 (Kyoto, Japan). Anti-involucrin (clone SY5) and anti-SREBP1 (clone 2A4) monoclonal 21 antibodies and an anti-filaggrin polyclonal antibody were obtained from Abcam 22 (Cambridge, UK). An anti-occludin monoclonal antibody (clone OC-3F10) and 23 anti-claudin-1 and anti-claudin-3 polyclonal antibodies were obtained from Invitrogen 24 (Camarillo, CA, USA). Anti-p38 MAPK antibody, anti-phospho p38 MAPK antibody 25 and phospho ERK1/2 antibody were obtained from Cell Signaling Technology (Danvers, 26 MA, USA). An anti-GAPDH polyclonal antibody was purchased from Santa Cruz 27 Biotechnology (Dallas, TX, USA). Polyvinylidene difluoride (PVDF) membrane and 28 enhanced chemiluminescence prime Western blotting detection reagent were purchased 29 from GE Healthcare (Buckinghamshire, UK). A Millicell ERS-2 System was obtained 30 from $\quad$ Millipore (Eschborn, Germany). MTS 31 [3-(4,5-dimethylthiazol-2-yl)-5-(3-carboxymethoxyphenyl)-2-(4-sulfophenyl)-2H-tetraz 32 olium] solution was obtained from Promega (Madison, WI, USA). PD98059 was 
1 obtained from Cayman Chemical (Ann Arbor, MI, USA). LY294002 was purchased 2 from Jena Bioscience (Jena, Germany). SB203580 was obtained from Med Chem 3 Express (Princeton, NJ, USA).

\section{Cell culture} supplemented with $10 \%$ fetal bovine serum, $100 \mathrm{U} / \mathrm{ml}$ penicillin, and $100 \mathrm{U} / \mathrm{ml}$ streptomycin in an atmosphere of $5 \% \mathrm{CO}_{2}$ and $95 \%$ air at $37^{\circ} \mathrm{C}$. NHEK were maintained in HuMedia-KG2 medium in an atmosphere of $5 \% \mathrm{CO}_{2}$ and $95 \%$ air at $37^{\circ} \mathrm{C}$. For in vitro differentiation assays, subconfluent keratinocytes were grown in CnT-07 growth medium for $48 \mathrm{~h}$. After reaching confluency, the medium was replaced with

11 CnT-02 differentiation medium supplemented with $1.8 \mathrm{mM} \mathrm{CaCl}_{2}$.

\section{Transepithelial electrical resistance (TER) measurement}

A HaCaT keratinocyte suspension $\left(1.8 \times 10^{5}\right.$ cells $)$ was seeded into a cell culture insert with a pore size of $0.4 \mathrm{~mm}$ and a growth area of $0.6 \mathrm{~cm}^{2}$, placed in a 24-well cell culture plate. Medium was added to the cell culture plate such that the level was equal to that inside the cell culture insert. Upon reaching confluency, HaCaT cells were differentiated as described above. Following induction of epithelial differentiation, TER was measured with a Millicell ERS-2 System every other day. Medium was replaced at the same time. Data represent the mean of five independent measurements.

\section{Western blotting}

21 HaCaT cells and NHEK were harvested in RIPA buffer to yield cell lysates. Cell 22 lysates were resolved by $10 \%$ sodium dodecyl sulfate polyacrylamide gel 23 electrophoresis and electrically transferred to a PVDF membrane. Subsequently, the 24 membrane was treated with blocking reagent (Tris-buffered saline containing $0.1 \%$ 25 Tween-20 and 5\% skimmed milk) for $2 \mathrm{~h}$ at room temperature. Blocked membranes 26 were incubated with primary antibodies (anti- involucrin (1:2000), anti-filaggrin 27 (1:1000), anti-SREBP1 (1:3000), anti-claudin-1 (1:2000), anti-claudin-3 (1:3000), 28 anti-occludin (1:3000), anti-p38 MAPK (1:2000), anti-phospho p38 MAPK (1:3000), 29 anti-phosphoERK1/2 (1:3000), anti-GAPDH (1:3000)), followed by incubation with a 30 horseradish peroxidase-conjugated secondary antibody (dilution ratio was 1:2000). 31 Immunoreactivity was detected using Western blotting detection reagents.

\section{Proliferation assay}


The proliferation of HaCaT cells in non-differentiating conditions was evaluated by 2 quantification of formazan produced by reduction of MTS by living cells by using a kit 3 (CellTiter 96® AQueous One Solution, Promega). HaCaT cells $(100 \mu \mathrm{l})$ were plated in 4 96-well plates. MTS solution $(20 \mu \mathrm{l})$ was added at the times indicated, and then cells were incubated at $37^{\circ} \mathrm{C}$ for $60 \mathrm{~min}$. The amount of formazan was measured by colorimetric quantification (absorbance at $490 \mathrm{~nm}$ ) using an iMark micro plate reader (Bio-Rad, Hercules, CA, USA). Results are expressed as means \pm standard deviations.

\section{Statistical analysis}

Data were analyzed by IBM SPSS Statistics (version 21) using the Student's t-test. The limit for statistical significance was set at $\mathrm{P}<0.05$.

\section{Results}

\section{Effect of lactoferrin on TER}

We investigated whether lactoferrin can promote the barrier function of differentiating keratinocytes. After induction of differentiation, TER was measured every 2 days to evaluate the barrier function of $\mathrm{HaCaT}$ cells. Bovine lactoferrin (1-10 $\mu \mathrm{M}$ ) was added to the differentiation medium at the onset of differentiation. A steady elevation of TER was observed in differentiating HaCaT cells in the absence of lactoferrin (Fig. 1). The elevation in TER was enhanced in lactoferrin-treated HaCaT cells, suggesting that lactoferrin improved the barrier function of differentiating keratinocytes (Fig. 1).

\section{Effect of lactoferrin on the expression of differentiation markers}

23 To evaluate the effects of lactoferrin on epithelial differentiation, we investigated 24 the expression of involucrin and filaggrin by Western blotting at 5 days after induction 25 of differentiation. When HaCaT cells were cultured in non-differentiating conditions $26\left(0.1 \mathrm{mM} \mathrm{CaCl}_{2}\right)$, a slight increase in involucrin and filaggrin expression was observed in 27 response to lactoferrin $(0.1-10 \mu \mathrm{M})$ (Fig. 2a). In HaCaT cells maintained in 28 differentiating conditions $\left(1.8 \mathrm{mM} \mathrm{CaCl}_{2}\right)$, expression of these differentiation markers 29 was higher than in non-differentiating conditions at all lactoferrin concentrations 30 ranging between 0.1 and $10 \mu \mathrm{M}$ (Fig. 2a). The increased expression of involucrin and 31 filaggrin was associated with the elevation of TER in differentiating conditions. This 32 observation suggests that lactoferrin promotes the barrier function of keratinocytes by 
1 promoting the differentiation and expression of cornified envelope components.

Next, we investigated the effect of lactoferrin on SREBP1 expression in HaCaT cells (Fig. 2b). Lactoferrin-induced expression of SREBP1 was observed even in non-differentiating conditions $\left(0.1 \mathrm{mM} \mathrm{CaCl}_{2}\right)$. It was detectable in cells treated with $0.1 \mu \mathrm{M}$ lactoferrin. When $\mathrm{HaCaT}$ cells were switched to differentiating conditions (1.8 $\mathrm{mM} \mathrm{CaCl}_{2}$ ), SREBP1 expression was detected even in the absence of lactoferrin. SREBP1 expression was more pronounced in cells treated with $10 \mu \mathrm{M}$ lactoferrin (Fig. $2 b)$.

In the epidermis, skin barrier function is mainly mediated by cornified envelope components. Tight junctions are another type of intracellular junction responsible for skin barrier function (Tebbe et al. 2002). Therefore, we investigated the effect of lactoferrin on expression of several tight junction components such as occludin, claudin-1, and claudin-3 (Fig. 2c). Claudin-1 and -3 are the major protein species present in the epidermis of healthy skin (Watson et al. 2007). In response to induction of epithelial differentiation $\left(1.8 \mathrm{mM} \mathrm{CaCl}_{2}\right)$, occludin expression was increased. However,

16 lactoferrin did not upregulate the expression of occludin or claudin-1 (Fig. 2c). 17 Interestingly, treatment of $\mathrm{HaCaT}$ cells with lactoferrin decreased expression of claudin-3 in both differentiating and non-differentiating conditions in a dose-dependent manner. These observations suggest that the promoting effects of bovine lactoferrin on

20 barrier function in keratinocytes are not mediated by elevation of the expression of tight 21 junction components.

22 Effect of lactoferrin on the differentiation of normal human epidermal 23 keratinocytes

24 The promoting effect of lactoferrin on epithelial differentiation was confirmed by 25 using normal human epidermal keratinocytes (NHEK). As HaCaT keratinocytes, 26 lactoferrin enhanced filaggrin and involucrin expression in NHEK maintained in 27 differentiating condition for 5 days (Fig. 2d). Lactoferrin-induced upregulation of 28 involucrin expression was also observed in NHEK maintained in non-differentiating 29 conditions $\left(0.1 \mathrm{mM} \mathrm{CaCl}_{2}\right)$.

30 Effect of lactoferrin on the proliferation of HaCaT keratinocytes

31 It is reported that loss of proliferative potential is associated with the 32 differentiation of keratinocytes. We performed the MTS colorimetric assay to evaluate 
1 the effect of bovine lactoferrin on $\mathrm{HaCaT}$ cell proliferation. The proliferation rate did 2 not differ in the presence or absence of bovine lactoferrin until day 2, whereas an 3 inhibitory effect of bovine lactoferrin on proliferation was observed at day 3 (Fig. 3).

4 The effect of bovine lactoferrin was dose-dependent at concentrations ranging between $5 \quad 1$ and $10 \mu \mathrm{M}$.

6 Effect of signaling inhibitors on lactoferrin-induced involucrin and filaggrin 7 expression

8 Next, we investigated the signaling molecules required for lactoferrin-induced 9 epithelial differentiation of HaCaT keratinocytes. Treatment with SB203580, a p38 10 mitogen-activated protein kinase (MAPK) $\alpha$ inhibitor, impaired lactoferrin-induced 11 upregulation of involucrin and filaggrin expression, whereas an inhibitory effect of 12 LY294002, a phosphoinositide 3-kinase (PI-3K) inhibitor, was observed only on 13 involucrin expression (Fig. 4a). PD98059, a MAPK kinase (MEK)/ERK1/2 inhibitor, 14 had no effect on involucrin or filaggrin expression. Inhibition of $\mathrm{p} 38 \mathrm{MAPK} \delta$ is toxic to 15 HaCaT keratinocytes; therefore, the effect of p38MAPK $\delta$ inhibitors on involucrin and 16 filaggrin expression was not carried out in the present study.

\section{Effect of lactoferrin in p38MAPK in differentiating keratinocytes}

18 To confirm the involvement of p38MAPK on lactoferrin-enhanced epithelial 19 differentiation, we investigated the time course of p38MAPK $\alpha$ and ERK1/2 20 phosphorylation in differentiating $\mathrm{HaCaT}$ cells (Fig. 4b), Un-differentiated cells 21 exhibited little p38MAPK $\alpha$ phosphorylation, along with increased phosphorylation at 22 day 4, followed by a return to baseline levels at day 6 . In the presence of lactoferrin, 23 enhanced $\mathrm{p} 38 \mathrm{MAPK} \alpha$ phosphorylation was observed in the initial stage of epithelial 24 differentiation (day 2). In contrast, ERK1/2 phosphorylation was observed in 25 un-differentiated $\mathrm{HaCaT}$ keratinocytes. Induction of epithelial differentiation resulted in 26 suppression of ERK1/2 phosphorylation in the presence or absence of lactoferrin. These 27 observations suggest that lactoferrin promotes epithelial differentiation by a 28 p38MAPK $\alpha$-dependent mechanism.

29

30

\section{Discussion}

32 The results of this study indicate that bovine lactoferrin promotes the elevation of 
1 TER in differentiating keratinocytes (Fig. 1). TER reflects the permeability of cell 2 layers to ions, with a higher TER indicating a lower ionic permeability (Francis et al. 3 1999). Therefore, our results suggested that bovine lactoferrin promotes the barrier 4 function of keratinocytes. In the epidermis, the barrier function is mainly mediated by 5 the cornified layer. Lactoferrin-induced elevation of TER was associated with increased 6 expression of cornified envelope components such as involucrin and filaggrin (Fig. 2a).

7 In differentiating (high calcium) conditions, expression of involucrin and filaggrin was 8 observed even when the lactoferrin concentration was relatively low $(0.1 \mu \mathrm{M})$. In 9 non-differentiating conditions (low calcium), expression of the differentiation markers 10 was low. This observation indicates that expression of the differentiation markers is 11 tightly regulated by extracellular calcium concentrations. The role of lactoferrin in 12 keratinocyte differentiation may reinforce the calcium-induced signaling pathway 13 required for keratinocyte differentiation.

14 Lactoferrin-induced expression of involucrin was blocked by SB203580, an 15 inhibitor of p38MAPKa (Fig. 4a). Many studies of the role of p38MAPK in 16 keratinocyte differentiation focus on $\mathrm{p} 38 \mathrm{MAPK} \delta$ (Eckert et al. 2003). Many agents that 17 promote involucrin gene expression increase $\mathrm{p} 38 \mathrm{MAPK} \delta$ kinase activity in 18 keratinocytes (Eckert et al. 2003; Eckert et al. 2004; Cuenda et al. 2007). In addition, 19 p38MAPK $\alpha$ also plays an important role in involucrin gene expression and keratinocyte 20 differentiation. Inhibition of p38MAPK $\alpha$ impairs involucrin gene expression induced by 21 treatment with methyl- $\beta$-cyclodextrin or ectopic expression of several MEKs in 22 keratinocytes (Cuenda et al. 2007; Dashti et al. 2001; Jans et al. 2004). Although the 23 mechanisms underlying lactoferrin-induced activation of p38MAPK $\alpha$ have not been 24 elucidated, lactoferrin is likely to enhance involucrin expression by a 25 p38MAPK $\alpha$-dependent mechanism. In addition, lactoferrin-induced enhancement of 26 involucrin expression was blocked by LY294002, a PI-3K inhibitor, suggesting that the 27 promoting effect of bovine lactoferrin on keratinocyte differentiation is also dependent 28 on the PI-3K signaling pathway (Fig. 4a). This result is consistent with previous studies 29 reporting that the activation of PI-3K in keratinocytes promotes growth arrest and 30 differentiation. Activation of PI-3K is observed at early stages of mouse keratinocyte 31 differentiation both in culture and in the intact epidermis in vivo (Calautti et al. 2005). 32 Inhibition of PI-3K blocks calcium-induced expression of involucrin in keratinocytes 
1 (Calautti et al. 2005; Xie et al. 2005). In this study, expression of SREBP1 was also 2 enhanced by treatment with lactoferrin (Fig. 2b). A previous report showed a role of 3 PI-3K in SREBP1 expression in HaCaT keratinocytes (Zhou et al. 2012). However, we 4 noted that lactoferrin-induced expression of SREBP1 was observed even in 5 non-differentiating (low calcium) conditions (Fig. 2b), suggesting that the mechanism 6 underlying lactoferrin-induced enhancement of SREBP1 expression is distinct from that 7 of involucrin and filaggrin. Further studies are required to elucidate the mechanisms by 8 which lactoferrin induces SREBP1.

In epidermis, involucrin is a major component of the cornified envelope, a structure synthesized at late stages of keratinocyte differentiation. Physical crosslinking of involucrin to other components of cornified envelope contributes to sustain epidermal barrier integrity. Lactoferrin-induced enhancement of involucrin expression suggests that the function of lactoferrin is mainly mediated by reinforcement of the cornified envelope. We did not observe a promoting effect of bovine lactoferrin on the expression of tight junction components (occludin, claudin-1). Claudin-3 expression was decreased in response to lactoferrin (Fig. 2c). Whereas epithelial barrier function is mainly sustained by cornified layer of epidermis, claudin-3 was only present in granular layer in epidermis (Watson et al. 2007). Therefore, this reduction is not likely to affect epithelial barrier function in $\mathrm{HaCaT}$ keratinocytes.

Lactoferrin exerts its biological activity by binding to its receptor(s)(Suzuki et al.

21 2005). Tang et al. reported that the promoting effect of human lactoferrin on the 22 migration of keratinocytes is dependent on low-density lipoprotein receptor-related 23 protein-1 (LRP-1) (Tang et al. 2010). LRP-1 acts as a lactoferrin receptor in fibroblasts, 24 osteoblasts, and adipocytes that converts the lactoferrin-binding signal into activation of 25 the ERK1/2 signaling pathway (Takayama et al. 2003; Grey et al. 2004; Ikoma-Seki et 26 al. 2015). In this study, lactoferrin-induced involucrin expression was not dependent on 27 ERK1/2 (Fig. 4). ERK1/2 is reportedly a negative regulator of involucrin expression 28 (Eckert et al. 2004). Therefore, a receptor(s) other than LRP-1 may be involved in the 29 promoting effect of lactoferrin on keratinocyte differentiation.

30 Defects in keratinocyte differentiation and skin barrier function cause 31 inflammatory skin diseases such as atopic dermatitis (Cork et al. 2009). The results of 32 this study suggest that lactoferrin contributes to host defense by increasing keratinocyte 
1 barrier function.

2

3

\section{Acknowledgments}

This work was supported by the Cross-ministerial Strategic Innovation Promotion Program (SIP), "Technologies for creating next-generation agriculture, forestry and fisheries" (ID: 14532924) from the Council for Science, Technology and Innovation (CSTI) (YT, RA, and AA), and a Grant-in-Aid for Scientific Research C (no. 25450413) from the Japan Society for the Promotion of Science (YT).

\section{References}

Actor, J.K., Hwang, S.A., and Kruzel, M.L. 2009. Lactoferrin as a natural immune modulator. Curr. Pharm. Des. 15(17):1956-1973. PMID: 19519436.

Calautti, E., Li, J., Saoncella, S., Brissette, J.L., and Goetinck, P.F. 2005.

17 Phosphoinositide3-kinase signaling to Akt promotes keratinocyte differentiation versus 18 death. J. Biol. Chem. 280(38):32856-32865. PMID: 16036919.

20 Cork, M.J., Danby, S.G., Vasilopoulos, Y., Hadgraft, J., Lane, M.E., Moustafa, M., Guy, 21 R.H., Macgowan, A.L., Tazi-Ahnini, R., and Ward, S.J. 2009. Epidermal barrier 22 dysfunction in atopic dermatitis. J. Invest. Dermatol. 129(8):1892-1908. 23 doi:10.1038/jid.2009.133. PMID: 19494826.

25 Cuenda, A., and Rousseau, S. 2007. p38 MAP-kinases pathway regulation, function and 26 role in human diseases. Biochim. Biophys. Acta. 1773(8):1358-1375. PMID: 17481747.

28 Dashti, S.R., Efimova, T., and Eckert, R.L. 2001. MEK6 regulates human involucrin 29 gene expression via a p38alpha - and p38delta -dependent mechanism. J. Biol. Chem. 30 276(29):27214-27220. PMID: 11454875.

31

32 Eckert, R.L., Efimova, T., Balasubramanian, S., Crish, J.F., Bone, F., and Dashti, S. 
1 2003. p38 Mitogen-activated protein kinases on the body surface--a function for p38

2 delta. J. Invest. Dermatol. 120(5):823-828. PMID: 12713588.

3

4 Eckert, R.L., Crish, J.F., Efimova, T., Dashti, S.R., Deucher, A., Bone, F., Adhikary, G.,

5 Huang, G., Gopalakrishnan, R., and Balasubramanian, S. 2004. Regulation of involucrin

6 gene expression. J. Invest. Dermatol. 123(1):13-22. PMID:15191537.

7

8 Engelmayer, J., Blezinger, P., and Varadhachary, A. 2008. Talactoferrin stimulates

9 wound healing with modulation of inflammation. J. Surg. Res. 149(2):278-286.

10 doi:10.1016/j.jss.2007.12.754. PMID: 18619616.

11

12 Francis, S.A., Kelly, J.M., McCormack, J., Rogers, R.A., Lai, J., Schneeberger, E.E.,

13 and Lynch, R.D. 1999. Rapid reduction of MDCK cell cholesterol by

14 methyl-beta-cyclodextrin alters steady state transepithelial electrical resistance. Eur. J.

15 Cell Biol. 78(7):473-484. PMID: 10472800.

16

17 Goldstein, J.L., DeBose-Boyd, R.A., and Brown, M.S. 2006. Protein sensors for 18 membrane sterols. Cell, 124(1):35-46. PMID: 16413480.

19

20 Grey, A., Banovic, T., Zhu, Q., Watson, M., Callon, K., Palmano, K., Ross, J., Naot, D., 21 Reid, I.R., and Cornish, J. 2004. The low-density lipoprotein receptor-related protein 1 22 is a mitogenic receptor for lactoferrin in osteoblastic cells. Mol. Endocrinol. 23 18(9):2268-2278. PMID: 15178744.

24

25 Ikoma-Seki, K., Nakamura, K., Morishita, S., Ono, T., Sugiyama, K., Nishino, H., 26 Hirano, H., and Murakoshi, M. 2015. Role of LRP1 and ERK and cAMP Signaling 27 Pathways in Lactoferrin-Induced Lipolysis in Mature Rat Adipocytes. PLoS One, 28 10(10):e0141378. doi: 10.1371/journal.pone.0141378. PMID: 26506094.

29

30 Jans, R., Atanasova, G., Jadot, M., and Poumay, Y. 2004. Cholesterol depletion 31 upregulates involucrin expression in epidermal keratinocytes through activation of $\mathrm{p} 38$. 32 J. Invest. Dermatol. 123(3):564-573. PMID: 15304097. 
1

2 Legrand, D., Elass, E., Carpentier, M., and Mazurier, J. 2005. Lactoferrin: a modulator 3 of immune and inflammatory responses. Cell. Mol. Life Sci. 62(22):2549-2559. PMID: 416261255.

5

6 7

Lyons, T.E., Miller, M.S., Serena, T., Sheehan, P., Lavery, L., Kirsner, R.S., Armstrong, D.G., Reese, A., Yankee, E.W., and Veves, A. 2007. Talactoferrin alfa, a recombinant human lactoferrin promotes healing of diabetic neuropathic ulcers: a phase $1 / 2$ clinical study. Am. J. Surg. 193(1):49-54. PMID: 17188087.

Micallef, L., Belaubre, F., Pinon, A., Jayat-Vignoles, C., Delage, C., Charveron, M., and Simon, A. 2009. Effects of extracellular calcium on the growth-differentiation switch in immortalized keratinocyte HaCaT cells compared with normal human keratinocytes. Exp. Dermatol. 18(2):143-151. doi: 10.1111/j.1600-0625.2008.00775.x. PMID: 18637039.

Sivamani, R.K., Garcia, M.S., and Isseroff, R.R. 2007. Wound re-epithelialization: modulating keratinocyte migration in wound healing. Front. Biosci.12:2849-2868. PMID: 17485264.

Suzuki, Y.A., Lopez, V., and Lönnerdal, B. 2005. Mammalian lactoferrin receptors: structure and function. Cell Mol. Life Sci. 62(22):2560-2575. PMID:16261254.

Takayama, Y., Takahashi, H., Mizumachi, K., and Takezawa, T. 2003. Low density lipoprotein receptor-related protein (LRP) is required for lactoferrin-enhanced collagen gel contractile activity of human fibroblasts. J. Biol. Chem. 278(24):22112-22118. PMID: 12672816.

Takayama, Y., and Aoki, R. 2012. Roles of lactoferrin on skin wound healing. Biochem. Cell Biol. 90(3):497-503. doi: 10.1139/o11-054. PMID: 22332789.

1

Tang, L., Wu, J.J., Ma, Q., Cui, T., Andreopoulos, F.M., Gil, J., Valdes, J., Davis, S.C., 
1 and $\mathrm{Li}$, J. 2010. Human lactoferrin stimulates skin keratinocyte function and wound

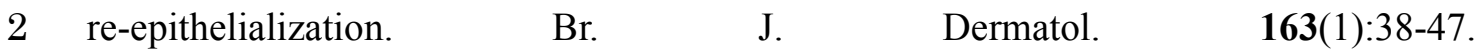
3 doi:10.1111/j.1365-2133.2010.09748.x. PMID: 20222924.

4

5 Tebbe, B., Mankertz, J., Schwarz, C., Amasheh, S., Fromm, M., Assaf, C., 6 Schultz-Ehrenburg, U., Sánchez Ruderish, H., Schulzke, J.D., and Orfanos, C.E. 2002.

7 Tight junction proteins: a novel class of integral membrane proteins. Expression in 8 human epidermis and in HaCaT keratinocytes. Arch. Dermatol. Res. 294(1-2):14-18. 9 PMID: 12071155.

11 Watson, R.E., Poddar, R., Walker, J.M., McGuill, I., Hoare, L.M., Griffiths, C.E., and 12 O'neill, C.A. 2007. Altered claudin expression is a feature of chronic plaque psoriasis. J. 13 Pathol. 212(4):450-458. PMID: 17582238.

15 Xie, Z., Singleton, P.A., Bourguignon, L.Y., and Bikle, D.D. 2005. Calcium-induced 16 human keratinocyte differentiation requires src- and fyn-mediated phosphatidylinositol 17 3-kinase-dependent activation of phospholipase C-gamma1. Mol. Biol. Cell, 18 16(7):3236-3246. PMID: 15872086.

20 Yokoyama, A., Makishima, M., Choi, M., Cho, Y., Nishida, S., Hashimoto, Y., and Terui, 21 T. 2009. Induction of SREBP-1c mRNA by differentiation and LXR ligand in human 22 keratinocytes. J. Invest. Dermatol. 129(6):1395-1401. doi:10.1038/jid.2009.15. PMID: 2319242521.

25 Zhou, B.R., Huang, Q.H., Xu, Y., Wu, D., Yin, Z.Q., and Luo, D. 2012. 26 Dihydrotestosterone induces SREBP-1 expression and lipogenesis through the 27 phosphoinositide 3-kinase/Akt pathway in HaCaT cells. Lipids Health Dis. 11:156. 28 doi:10.1186/1476-511X-11-156. PMID: 23153363.

29 
1 Figure legends

2 Fig. 1. Effect of bovine lactoferrin on the transepithelial electrical resistance (TER) of

3 differentiating HaCaT cells. After induction of epithelial differentiation, HaCaT cells

4 were mock-treated (open circles) or treated with lactoferrin $(1 \mu \mathrm{M}$ : filled circles, $5 \mu \mathrm{M}$ :

5 open triangles, $10 \mu \mathrm{M}$ : filled squares) for the indicated amount of time. Error bars

6 represent the mean \pm standard deviation. $\mathrm{n}=3$ in five independent experiments.

$7 \quad * * \mathrm{P}<0.01$ and $* \mathrm{P}<0.05$ versus mock-treated cells.

8

9 Fig. 2. Effect of bovine lactoferrin on epithelial differentiation of keratinocytes. (a)

10 Expression of filaggrin and involucrin in $\mathrm{HaCaT}$ keratinocytes. Confluent $\mathrm{HaCaT}$ cells

11 were allowed to differentiate $\left(1.8 \mathrm{mM} \mathrm{CaCl}_{2}\right)$ for 5 days or were cultured in

12 non-differentiating conditions $\left(0.1 \mathrm{mM} \mathrm{CaCl}_{2}\right)$ for the same amount of time. The

13 indicated concentrations of bovine lactoferrin were added to the culture media. (b)

14 Expression of sterol regulatory element-binding protein-1 (SREBP1). (c) Expression of

15 tight junction proteins. GAPDH was used as a loading control. (d) Expression of

16 filaggrin and involucrin in normal human epidermal keratinocytes. Experimental

17 procedure is same as for HaCaT keratinocytes.

19 Fig. 3. Effect of bovine lactoferrin on the proliferation of HaCaT keratinocytes. HaCaT

20 cells were mock-treated or treated with bovine lactoferrin $(1-10 \mu \mathrm{M})$ for the indicated

21 amount of time. Relative numbers of HaCaT cells were assessed by measuring

22 absorbance at $490 \mathrm{~nm}$, which corresponds to formazan production by living cells. Error

23 bars represent the mean \pm standard deviation. $* * \mathrm{P}<0.01$ versus mock-treated cells.

25 Fig. 4. The involvement of the p38MAPK signaling pathways in lactoferrin-induced 26 differentiation of $\mathrm{HaCaT}$ keratinocytes. (a) HaCaT cells were differentiated in the 27 presence or absence of $10 \mu \mathrm{M}$ bovine lactoferrin for 5 days. PD98059 $(40 \mu \mathrm{M})$, 28 SB203580 $(10 \mu \mathrm{M})$, or LY294002 $(10 \mu \mathrm{M})$ were added at the same time. Cell 29 differentiation was evaluated by the expression levels of involucrin and filaggrin. 30 GAPDH was used as a loading control. (b) Effect of lactoferrin on phosphorylation of 31 p38MAPK and ERK1/2 in differentiating HaCaT cells. HaCaT cells were differentiated 32 in the presence or absence of $10 \mu \mathrm{M}$ bovine lactoferrin for the indicated times. 


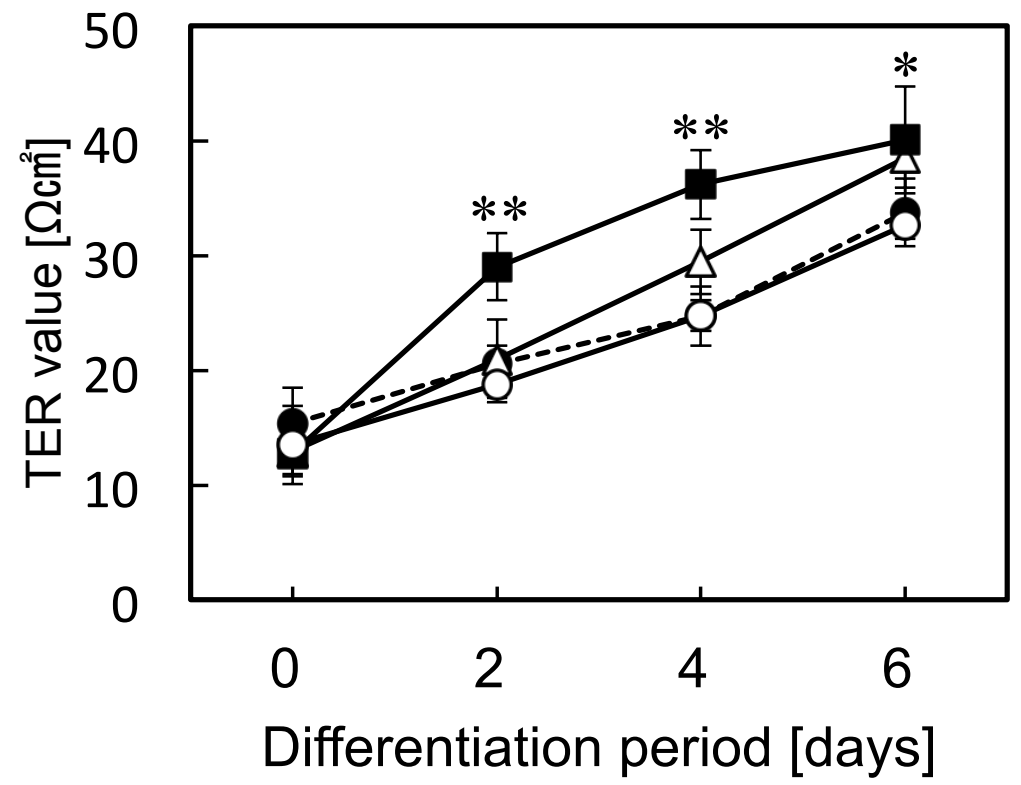

Fig.1 Uchida ethatss;//mc06.manuscriptcentral.com/bcb-pubs 
a

\begin{tabular}{c|c|c|c|c|c|c|c|c}
\hline $\mathrm{Ca}^{2+}[\mathrm{mM}]$ & \multicolumn{4}{|c|}{0.1} & \multicolumn{5}{c}{1.8} \\
\hline Lactoferrin & 0 & 0.1 & 1.0 & 10 & 0 & 0.1 & 1.0 & 10 \\
\hline
\end{tabular}

Involucrin

Filaggrin

GAPDH

b

\begin{tabular}{|c|c|c|c|c|c|c|c|c|}
\hline $\mathrm{Ca}^{2+}[\mathrm{mM}]$ & \multicolumn{4}{|c|}{0.1} & \multicolumn{4}{|c|}{1.8} \\
\hline Lactoferrin & 0 & 0.1 & 1.0 & 10 & 0 & 0.1 & 1.0 & 10 \\
\hline SREBP1 & \multicolumn{8}{|c|}{$== \pm \equiv=2$} \\
\hline GAPDH & & & & & & & & \\
\hline
\end{tabular}

C

\begin{tabular}{c|c|c|c|c|c|c|c|c}
\hline $\mathrm{Ca}^{2+}[\mathrm{mM}]$ & \multicolumn{4}{|c|}{0.1} & \multicolumn{5}{c}{1.8} \\
\hline Lactoferrin & 0 & 0.1 & 1.0 & 10 & 0 & 0.1 & 1.0 & 10 \\
{$[\mu \mathrm{M}]$}
\end{tabular}

Claudin-1

Claudin-3

Occludin

GAPDH

d

\begin{tabular}{l|c|c|c|c}
\hline $\mathrm{Ca}^{2+}[\mathrm{mM}]$ & \multicolumn{2}{|c|}{0.1} & \multicolumn{2}{|c}{1.8} \\
\hline Lactoferrin & 0 & 10 & 0 & 10 \\
\hline
\end{tabular}

Involucrin

Filaggrin

GAPDH 
(A490)

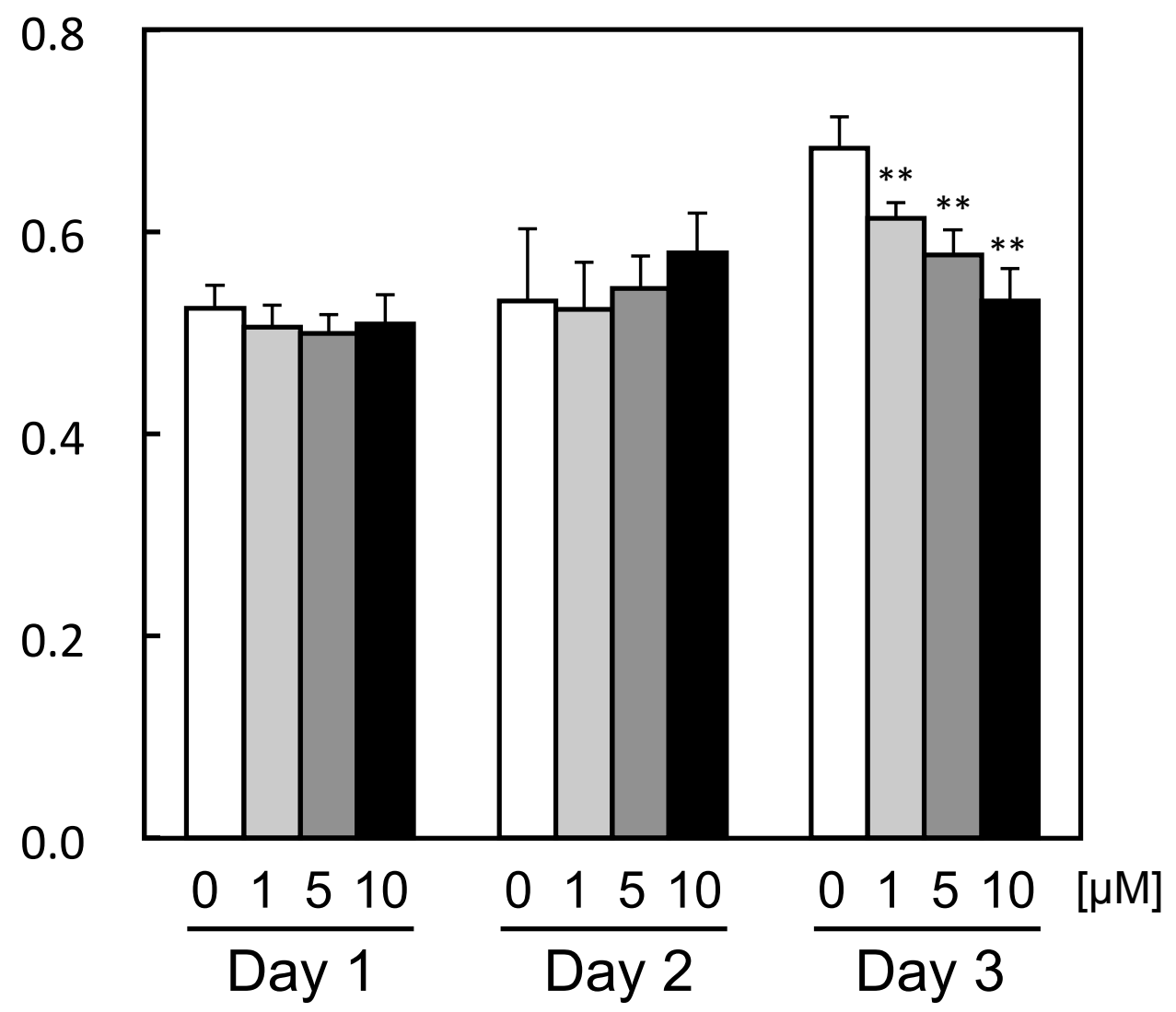

Fig.3 Uchida et al., 


a \begin{tabular}{c|c|c|c|c|c|c|c|c}
\hline Lactoferrin & \multicolumn{5}{|c|}{0} & \multicolumn{3}{|c}{10} \\
\hline PD98059 & - & + & - & - & - & + & - & - \\
\hline SB203580 & - & - & + & - & - & - & + & - \\
\hline LY294002 & - & - & - & + & - & - & - & + \\
\hline
\end{tabular}

Filaggrin

GAPDH
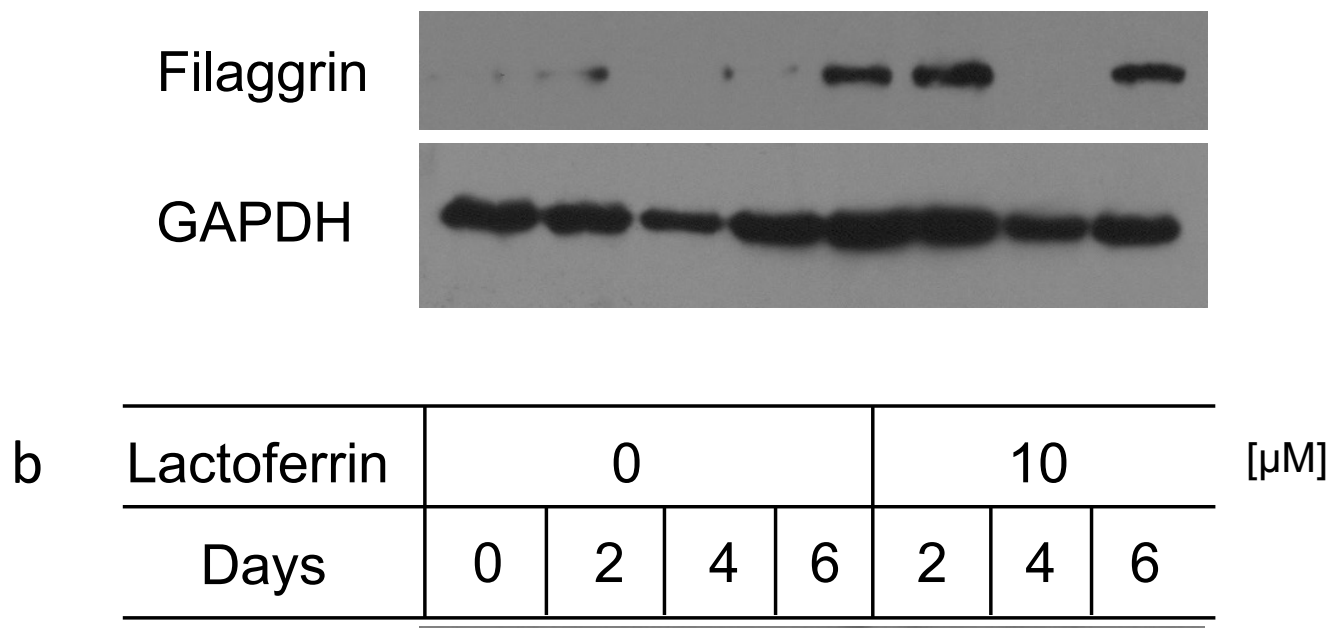

p-p38MAPK

p-ERK1/2

p38MAPK

Fig.4 Uchida et al., 